\title{
Characteristics Analysis of Suspending Force for Hybrid Stator Bearingless SRM
}

\author{
Jin-Woo Ahn ${ }^{\dagger}$ and Dong-Hee Lee*
}

\begin{abstract}
In this paper, a characteristics analysis and calculation of the suspending force of a novel bearingless switched reluctance motor (BLSRM) with hybrid stator poles is proposed. The operating principle and permeance are calculated to find an appropriate control scheme for a proposed motor. Furthermore, a mathematical model for suspending force is derived. Finite element analysis is also employed to compare with the expressions for suspending force. Finally, the validity of the structure and the mathematical model is verified by simulation results.
\end{abstract}

Keywords: Bearingless SRM, Hybrid pole, Permeance model, FEM analysis, Suspending force

\section{Introduction}

In modern industrial applications, high-speed machines such as molecular pumps, centrifuges, and compressors are frequently used. Switched reluctance motor (SRM) is a double-salient, single-excited motor. The stator consists of simple concentric windings, while the rotor has no windings or permanent magnets. Therefore, SRM is suitable for high-speed machines. However, many problems occur when traditional mechanical bearings are used to bear the shaft of high-speed or ultra-high-speed machines. For instance, the high-speed rotation of the rotor causes the increase in the frictional drag and serious exacerbation of bearing abrasion. This not only lowers the efficiency of the machine and reduces the service life of bearings, but also increases the maintenance burden for machines and bearings.

In order to solve these problems, studies have been recently made on various bearingless switched reluctance motors (BLSRMs). To overcome the disadvantages [1]-[3], a novel BLSRM structure with hybrid stator poles is proposed. An 8/10 BLSRM prototype is designed and analyzed. A calculation model for suspending force is also developed in order to carry out real-time control of the BLSRM. Significant fringing flux between the stator and the rotor is considered in the model, and eccentric errors in $\alpha$ and $\beta$ directions are taken into account. The validity of the structure and the calculation model is verified through finite element analysis (FEA) and simulation results.

\section{Structure and Principles}

\subsection{Conventional BLSRM}

Fig. 1 shows the structure and operating principle of a

\footnotetext{
$\dagger \quad$ Corresponding Author: Department of Mechatronics Engineering, Kyungsung University, Korea. (jwahn@ks.ac.kr)

* Department of Mechatronics Engineering, Kyungsung University, Korea. (leedh@ks.ac.kr)

Received: June 3, 2010; Accepted: November 2, 2010
}

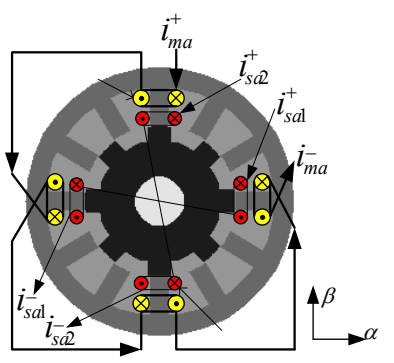

(a) Basic structure

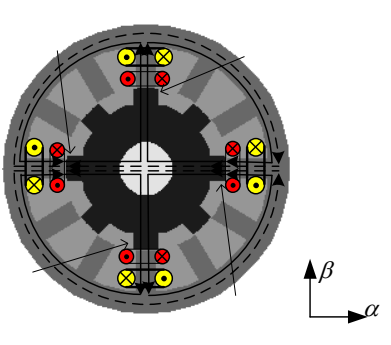

(b) Operating principle
Fig. 1. Conventional BLSRM.

conventional BLSRM. In conventional BLSRM, it should be noted that the four-pole flux produced by the currents of the torque winding is essential for suspension force generation. This also means that suspending force cannot be produced if no currents flow through the torque winding.

The curves of inductance, suspending force, and torque can be obtained through FEM and are shown in Fig. 2. As can be seen from the figure, the region for generating torque is from $\theta_{1}$ to $\theta_{3}$, whereas that for generating suspending force is from $\theta_{2}$ to $\theta_{4}$. The overlap region between generating torque and generating suspending force is from $\theta_{2}$ to $\theta_{3}$. Ideally, it is best for the motor to operate in this overlap region, in which enough torque and suspending force can be generated simultaneously. However, because of the inherent principle of torque and suspending force in the SRM, this overlap region is very narrow. In addition, an operating point has to be selected to compromise between the torque and suspending force when using the conventional structure. Accordingly, regions for generating torque and suspending force cannot be fully utilized. Therefore, in order to get sufficient suspending force, the current must be increased and the dwell angle should be moved toward an aligned position. This will result in high copper loss and large negative torque. Hence, efficiency is reduced, thermal load is increased, and speed is limited. 


\subsection{Proposed BLSRM}

Fig. 3(a) shows the structures of the proposed BLSRM. As can be seen from the figure, the structure is different from the conventional one since it has two types of stator poles on the stator. One is torque pole, such as A1, A2, B1, and B2, which mainly produces torque, while the other is suspending force pole, such as $\mathrm{P}_{\mathrm{x} 1}, \mathrm{P}_{\mathrm{x} 2}, \mathrm{P}_{\mathrm{x} 3}$, and $\mathrm{P}_{\mathrm{x} 4}$, which mainly generates suspending force to suspend rotor and shaft. Additionally, the pole arc of the suspending force pole is one pole pitch of the rotor for producing continuous suspending force. The windings on poles $\mathrm{A} 1$ and $\mathrm{A} 2$ are connected in a series to construct torque winding $\mathrm{A}$, while the windings on poles $\mathrm{B} 1$ and $\mathrm{B} 2$ are connected in a series to construct torque winding $\mathrm{B}$. The windings on poles $\mathrm{P}_{\mathrm{x} 1}$, $\mathrm{P}_{\mathrm{x} 2}, \mathrm{P}_{\mathrm{x} 3}$, and $\mathrm{P}_{\mathrm{x} 4}$ are independently controlled to construct four suspending force windings - P1, P2, P3, and P4 - in the $\mathrm{x}$ and $\mathrm{y}$ directions.

Fig. 3(b) shows the control principle of suspending force. From this figure, it can be seen that when the rotor has eccentric displacement in the positive y direction, only current $i_{2}$ will be turned on and the other suspending force windings $\mathrm{P}_{\mathrm{x} 1}, \mathrm{P}_{\mathrm{x} 2}$, and $\mathrm{P}_{\mathrm{x} 3}$ are turned off. Accordingly, the suspending force in the negative y direction is generated.
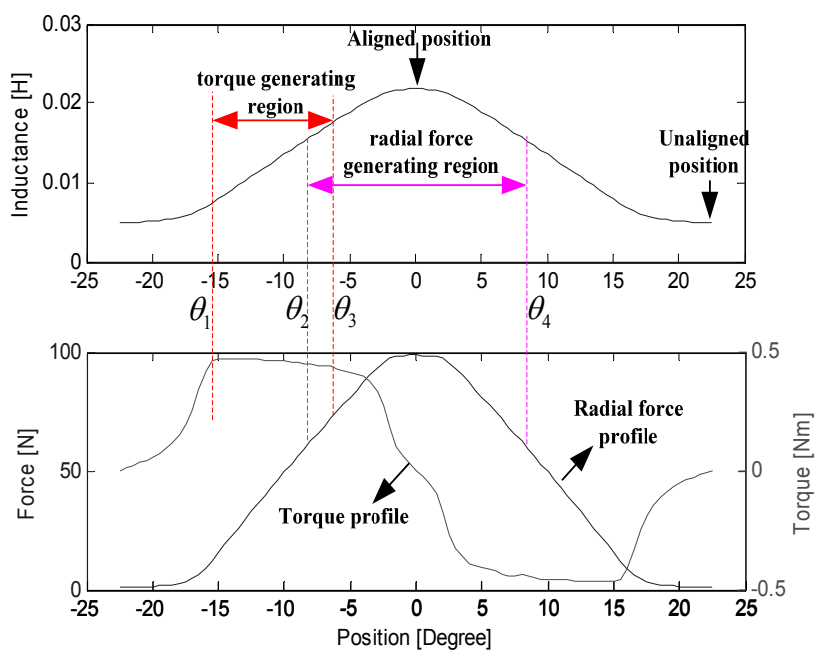

Fig. 2. Inductance, torque, and suspending force profiles of a conventional BLSRM.

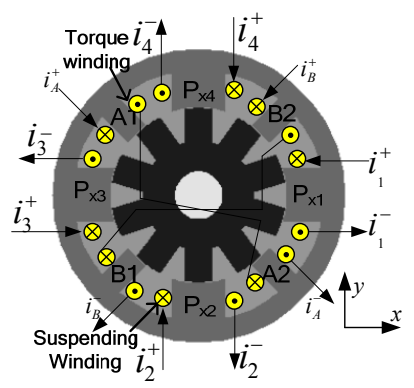

(a) Proposed BLSRM

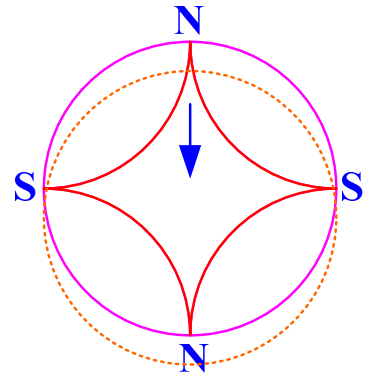

(b) Suspending control principle
Fig. 3. Structure and control principle of the proposed BLSRM.
Current $\mathrm{i}_{2}$ can be regulated until the rotor is in a balanced position. Using the same method, if the rotor has eccentric displacement in the positive $\mathrm{x}$ direction at the same time, only winding $P_{x 3}$ needs to be turned on and current $i_{3}$ is regulated to enable the rotor to return to its zero eccentric position.

\subsection{Calculation Model of Suspending Force}

The assumptions are as follows:

- Magnetic circuit saturation can be neglected.

- Leakage flux is ignored.

- Axial eccentricity is small compared with the length of the air gap.

In the proposed structure, the width of the pole arc of the rotor is $\beta_{\mathrm{r}}$, while that of the stator for generating suspending force is one pole pitch of rotor $\tau_{\mathrm{r}}$. At the same time, $\beta_{0}$ is defined as $1 / 2\left(\tau_{\mathrm{r}}-\beta_{\mathrm{r}}\right)$. When the relation between $\beta_{0}$ and the sum of the rotor angle $\theta$ and displacement $\beta$ in the $y$ direction is different, the model of magnetic conductance is also different. In Figs. 4(a) and 4(b), the magnetic field distribution of two cases with $\mathrm{r} \theta+\beta \leq \beta_{0}$ and $\beta_{0} \leq \mathrm{r} \theta+\beta \leq$ $\left(\beta_{0}+0.5 \beta_{\mathrm{r}}\right)$ are shown, respectively.

Based on Fig. 4, simplified equivalent magnetic circuits for the two cases are shown in Fig. 5.

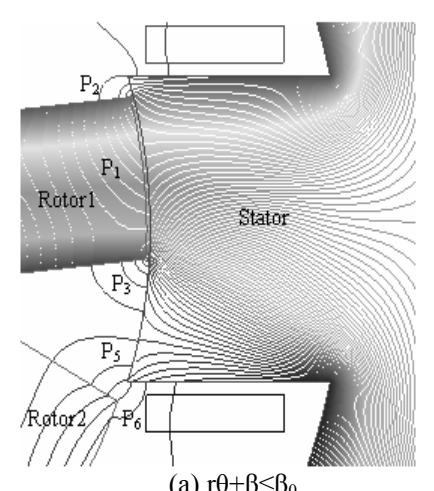

(a) $r \theta+\beta \leq \beta_{0}$

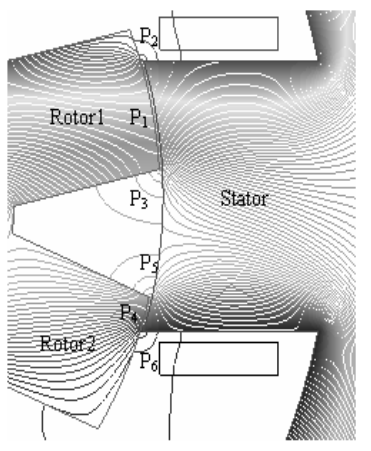

(b) $\beta_{0} \leq \mathrm{r} \theta+\beta \leq\left(\beta_{0}+0.5 \beta_{\mathrm{r}}\right)$
Fig. 4. Magnetic field distribution of air gap.

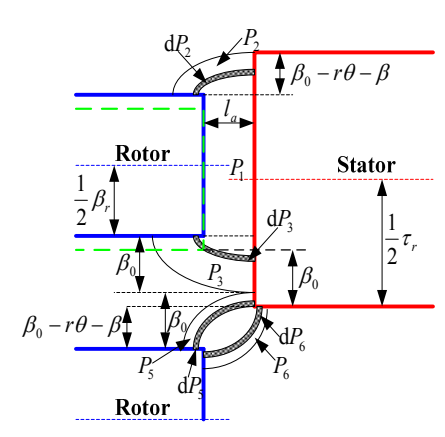

(a) $r \theta+\beta \leq \beta_{0}$

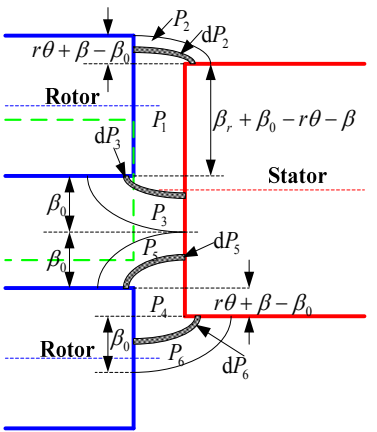

(b) $\beta_{0} \leq \mathrm{r} \theta+\beta \leq\left(\beta_{0}+0.5 \beta_{\mathrm{r}}\right)$
Fig. 5. Simplified equivalent magnetic circuit of air gap. 


\section{1) Case 1: $r \theta+\beta \leq \beta_{0}$}

In Fig. 5(a), the magnetic conductance can be divided into five parts. The overlap part $P_{1}$ between the stator and the rotor can be simplified as a linear magnetic circuit. Other parts such as $\mathrm{P}_{2}, \mathrm{P}_{3}, \mathrm{P}_{4}$, and $\mathrm{P}_{5}$ can be considered as elliptical magnetic circuits. Therefore, $\mathrm{P}_{1}$ can be described by the following equation [4]:

$$
P_{1}=\int_{0}^{\beta_{r}} \frac{\mu_{0} l \mathrm{~d} x}{l_{0}-\alpha+\frac{\beta \theta}{2}}=\frac{\mu_{0} l \beta_{r}}{l_{0}-\alpha+\frac{\beta \theta}{2}}
$$

where $l$ is the axial length of the core; $\mu_{0}$ is the permeability; $l_{0}$ is the length of the air gap without eccentricity; and $\alpha$ is the eccentric displacement in the $\mathrm{x}$ axis direction.

From Fig. 5(a), it can be seen that the solvable ranges of $\mathrm{P}_{2}, \mathrm{P}_{3}, \mathrm{P}_{5}$, and $\mathrm{P}_{6}$ are $\left[0, \beta_{0}-\mathrm{r} \theta-\beta\right],\left[0, \beta_{0}\right],\left[\beta_{0}-\mathrm{r} \theta-\beta, \beta_{0}\right]$, and $\left[\beta_{0}-\mathrm{r} \theta-\beta, \beta_{0}\right]$. Therefore, the corresponding magnetic conductance of these parts can be expressed as the following equations:

$$
\begin{aligned}
P_{2} & =\int_{0}^{\beta_{0}-r \theta-\beta} \frac{\mu_{0} l\left(a l_{a}+2 x\right) \mathrm{d} x}{\left(2 l_{a}+\pi x\right)\left(a l_{a}+x\right)} \\
& =\frac{\mu_{0} l}{a \pi-2}\left[\begin{array}{l}
a \ln \frac{a l_{a}-r \theta-\beta+\beta_{0}}{a l_{a}}+ \\
\frac{a \pi-4}{\pi} \ln \frac{2 l_{a}+\pi\left(-r \theta-\beta+\beta_{0}\right)}{2 l_{a}}
\end{array}\right] \\
P_{3} & =\int_{0}^{\beta_{0}} \frac{\mu_{0} l\left(a l_{a}+2 x\right) \mathrm{d} x}{\left(2 l_{a}+\pi x\right)\left(a l_{a}+x\right)} \\
& =\frac{\mu_{0} l}{a \pi-2}\left[\begin{array}{l}
a \ln \frac{a l_{a}+\beta_{0}}{a l_{a}}+\frac{a \pi-4}{\pi} \ln \frac{2 l_{a}+\pi \beta_{0}}{2 l_{a}}
\end{array}\right] \\
P_{5} & =P_{6}=\int_{\beta_{0}-r \theta-\beta}^{\beta_{0}} \frac{\mu_{0} l\left(a l_{a}+2 x\right) \mathrm{d} x}{\left(2 l_{a}+\pi x\right)\left(a l_{a}+x\right)} \\
& =\frac{\mu_{0} l}{a \pi-2}\left[\begin{array}{l}
a \ln \frac{a l_{a}+\beta_{0}}{a l_{a}}+\frac{a \pi-4}{\pi} \ln \frac{2 l_{a}+\pi \beta_{0}}{2 l_{a}} \\
-a \ln \frac{a l_{a}-r \theta-\beta+\beta_{0}}{a l_{a}}- \\
\frac{a \pi-4}{\pi} \ln \frac{2 l_{a}+\pi\left(-r \theta-\beta+\beta_{0}\right)}{2 l_{a}}
\end{array}\right]
\end{aligned}
$$

where $\mathrm{a}$ is the coefficient acquired through the FEM analysis, and $l_{a}$ is described as (5) [5]:

$$
l_{a}=l_{0}-\alpha+\frac{\beta \theta}{2}
$$

Thus, all magnetic conductance of air gap $\mathrm{P}_{\mathrm{x} 1}$ is shown in (6).

\section{2) Case 2: $\beta_{0} \leq r \theta+\beta \leq\left(\beta_{0}+0.5 \beta_{r}\right)$}

From Fig. 5(b), the magnetic conductance can be divided into six parts. The overlap parts between the stator and the rotor $-\mathrm{P}_{1}$ and $\mathrm{P}_{4}$ - can be simplified as linear magnetic circuits. Other parts can be considered as elliptical magnetic circuits. The solvable ranges from $P_{1}$ to $P_{6}$ are $[0$, $\left.\beta_{\mathrm{r}}+\beta_{0}-\mathrm{r} \theta-\beta\right],\left[0, \mathrm{r} \theta+\beta-\beta_{0}\right],\left[0, \beta_{0}\right],\left[0, \mathrm{r} \theta+\beta-\beta_{0}\right],\left[0, \beta_{0}\right]$, and $\left[0, \beta_{0}\right]$, respectively. Therefore, $\mathrm{P}_{1}$ and $\mathrm{P}_{4}$ can be described as (7)-(10).

$$
\begin{aligned}
& P_{x 1}=P_{1}+P_{2}+P_{3}+2 P_{5} \\
& =\frac{\mu_{0} l \beta_{r}}{l_{0}-\alpha+\frac{\beta \theta}{2}}+3 \frac{\mu_{0} l}{a \pi-2}\left[\begin{array}{l}
a \ln \frac{a l_{a}+\beta_{0}}{a l_{a}}+ \\
\frac{a \pi-4}{\pi} \ln \frac{2 l_{a}+\pi \beta_{0}}{2 l_{a}}
\end{array}\right] \\
& -\frac{\mu_{0} l}{a \pi-2}\left[\begin{array}{l}
a \ln \frac{a l_{a}-r \theta-\beta+\beta_{0}}{a l_{a}}+ \\
\frac{a \pi-4}{\pi} \ln \frac{2 l_{a}+\pi\left(-r \theta-\beta+\beta_{0}\right)}{2 l_{a}}
\end{array}\right] \\
& P_{1}=\int_{0}^{\beta_{r}+\beta_{0}-r \theta-\beta} \frac{\mu_{0} l \mathrm{~d} x}{l_{0}-\alpha+\frac{\beta \theta}{2}}=\frac{\mu_{0} l\left(\beta_{r}+\beta_{0}-r \theta-\beta\right)}{l_{0}-\alpha+\frac{\beta \theta}{2}} \\
& P_{2}=\int_{0}^{r \theta+\beta-\beta_{0}} \frac{\mu_{0} l\left(a l_{a}+2 x\right) \mathrm{d} x}{\left(2 l_{a}+\pi x\right)\left(a l_{a}+x\right)} \\
& =\frac{\mu_{0} l}{a \pi-2}\left[\begin{array}{l}
a \ln \frac{a l_{a}+r \theta+\beta-\beta_{0}}{a l_{a}}+ \\
\frac{a \pi-4}{\pi} \ln \frac{2 l_{a}+\pi\left(r \theta+\beta-\beta_{0}\right)}{2 l_{a}}
\end{array}\right] \\
& P_{4}=\int_{0}^{r \theta+\beta-\beta_{0}} \frac{\mu_{0} l \mathrm{l} x}{l_{0}-\alpha+\frac{\beta \theta}{2}}=\frac{\mu_{0} l\left(r \theta+\beta-\beta_{0}\right)}{l_{0}-\alpha+\frac{\beta \theta}{2}} \\
& P_{3}=P_{5}=P_{6}=\int_{0}^{\beta_{0}} \frac{\mu_{0} l\left(a l_{a}+2 x\right) \mathrm{d} x}{\left(2 l_{a}+\pi x\right)\left(a l_{a}+x\right)} \\
& =\frac{\mu_{0} l}{a \pi-2}\left[a \ln \frac{a l_{a}+\beta_{0}}{a l_{a}}+\frac{a \pi-4}{\pi} \ln \frac{2 l_{a}+\pi \beta_{0}}{2 l_{a}}\right]
\end{aligned}
$$

Hence, all the magnetic conductance of air gap $\mathrm{P}_{\mathrm{x} 1}$ is equal to the following:

$$
\begin{aligned}
& P_{x 1}=P_{1}+P_{2}+P_{3}+P_{4}+P_{5}+P_{6} \\
& =\frac{\mu_{0} l \beta_{r}}{l_{0}-\alpha+\frac{\beta \theta}{2}}+3 \frac{\mu_{0} l}{a \pi-2}\left[\begin{array}{l}
a \ln \frac{a l_{a}+\beta_{0}}{a l_{a}}+ \\
\frac{a \pi-4}{\pi} \ln \frac{2 l_{a}+\pi \beta_{0}}{2 l_{a}}
\end{array}\right] \\
& +\frac{\mu_{0} l}{a \pi-2}\left[\begin{array}{l}
a \ln \frac{a l_{a}+r \theta+\beta-\beta_{0}}{a l_{a}}+ \\
\frac{a \pi-4}{\pi} \ln \frac{2 l_{a}+\pi\left(r \theta+\beta-\beta_{0}\right)}{2 l_{a}}
\end{array}\right]
\end{aligned}
$$

From the symmetric structure of the BLSRM, the air gap permeance of $\mathrm{P}_{\mathrm{x} 2}, \mathrm{P}_{\mathrm{x} 3}$, and $\mathrm{P}_{\mathrm{x} 4}$ can be calculated using the same method. 


\subsection{Suspending Force Calculation}

Taking $\mathrm{P}_{\mathrm{X} 1}$ suspending winding as an example, the suspending forces in $\mathrm{x}$ direction $\mathrm{F}_{\alpha}$ and $\mathrm{y}$ direction $\mathrm{F}_{\beta}$ are deduced as the following method.

As the permeability of air gap is constant, permeance $\mathrm{P}_{\mathrm{x} 1}$, $\mathrm{P}_{\mathrm{x} 2}, \mathrm{P}_{\mathrm{x} 3}$, and $\mathrm{P}_{\mathrm{x} 4}$ are only related to the dimensions of the motor. The stored electromagnetic energy can be expressed as the following equation:

$$
W_{a}=\frac{1}{2} L i^{2}=\frac{1}{2} P(N i)^{2}
$$

where $i$ is the current; $\mathrm{P}$ is the permeance of air gap; and $\mathrm{N}$ is the winding turn.

Therefore, the energy at $\mathrm{P}_{\mathrm{x} 1}$ is as follow:

$$
W_{x 1}=\frac{1}{2} P_{x 1}(N i)^{2}
$$

Furthermore, suspending forces $\mathrm{F}_{\alpha}$ and $\mathrm{F}_{\beta}$ can be derived from the derivatives of energy with respect to displacement $\alpha, \beta$, and the angle of the rotor.

$$
\begin{aligned}
& F_{\alpha}=\frac{\partial W_{x 1}}{\partial \alpha}=\frac{1}{2} \frac{\partial P_{x 1}}{\partial \alpha}(N i)^{2} \\
& F_{\beta}=\frac{\partial W_{x 1}}{\partial \beta}=\frac{1}{2} \frac{\partial P_{x 1}}{\partial \beta}(N i)^{2}
\end{aligned}
$$

According to the symmetric structure, only period $\left[0, \beta_{0}\right.$ $\left.+0.5 \beta_{\mathrm{r}}\right]$ is considered, and the solution of the suspending force is also divided into two cases.

\section{1) Case 1: $r \theta+\beta \leq \beta_{0}$}

According to (6), (14), and (15), suspending forces in the $\mathrm{x}$ and $\mathrm{y}$ directions can be obtained as the following equations:

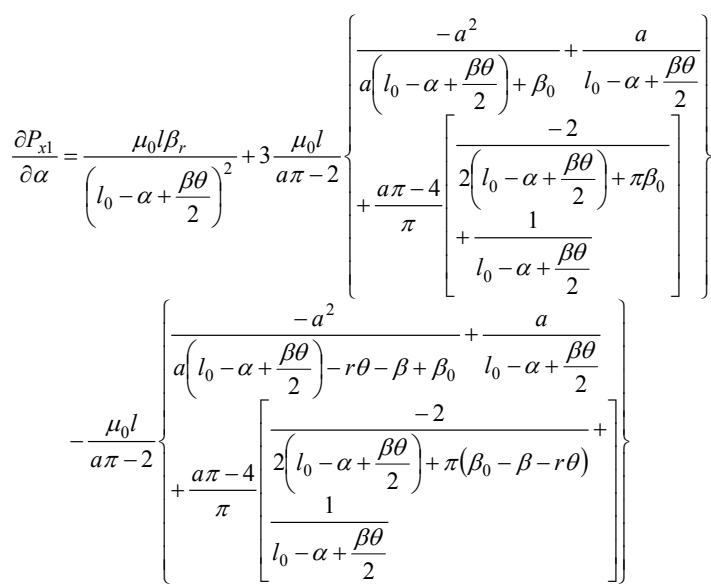

$$
\begin{aligned}
& \frac{\partial P_{x 1}}{\partial \beta} \approx \frac{-\mu_{0} l \beta_{r}}{\left(l_{0}-\alpha+\frac{\beta \theta}{2}\right)^{2}} \frac{\theta}{2}
\end{aligned}
$$

2) Case 2: $\beta_{0} \leq r \theta+\beta \leq\left(\beta_{0}+0.5 \beta_{r}\right)$

According to (11), (14), and (15), suspending forces in the $\mathrm{x}$ and $\mathrm{y}$ directions can be obtained as the following equations:

$$
\begin{aligned}
& \frac{\partial P_{x 1}}{\partial \alpha}=\frac{\mu_{0} l \beta_{0}}{\left(l_{0}-\alpha+\frac{\beta \theta}{2}\right)^{2}}+3 \frac{\mu_{0} l}{a \pi-2}\left\{\begin{array}{l}
\frac{-a^{2}}{a\left(l_{0}-\alpha+\frac{\beta \theta}{2}\right)+\beta_{0}}+\frac{a}{l_{0}-\alpha+\frac{\beta \theta}{2}} \\
+\frac{a \pi-4}{\pi}\left[\begin{array}{c}
\frac{-2}{2\left(l_{0}-\alpha+\frac{\beta \theta}{2}\right)+\pi \beta_{0}} \\
+\frac{1}{l_{0}-\alpha+\frac{\beta \theta}{2}}
\end{array}\right]
\end{array}\right\} \\
& +\frac{\mu_{0} l}{a \pi-2}\left\{\begin{array}{l}
\frac{-a^{2}}{a\left(l_{0}-\alpha+\frac{\beta \theta}{2}\right)+r \theta+\beta-\beta_{0}}+\frac{a}{l_{0}-\alpha+\frac{\beta \theta}{2}} \\
\left.+\frac{a \pi-4}{\pi}\left[\begin{array}{l}
\frac{-2}{2\left(l_{0}-\alpha+\frac{\beta \theta}{2}\right)+\pi\left(r \theta+\beta-\beta_{0}\right)} \\
+\frac{1}{l_{0}-\alpha+\frac{\beta \theta}{2}}
\end{array}\right]\right\} \\
\frac{\partial P_{x 1}}{\partial \beta} \approx \frac{\mu_{0} l}{\left(l_{0}-\alpha+\frac{\beta \theta}{2}\right)^{2}}\left[-\frac{\theta}{2}\left(2 \beta_{r}+\beta_{0}-2 r \theta-2 \beta\right)\right.
\end{array}\right\}
\end{aligned}
$$

\section{3) FEA Comparison}

The finite element method is applied to calculate the suspending force. Figs. 6 and 7 show the results of the comparison between the FEA and the permeance model. From these two figures, the values obtained from the calculation model are in good agreement with that of the FEA results. The error, from consumption of saturation, leakage flux, and fringing effects, is within $10 \%$.

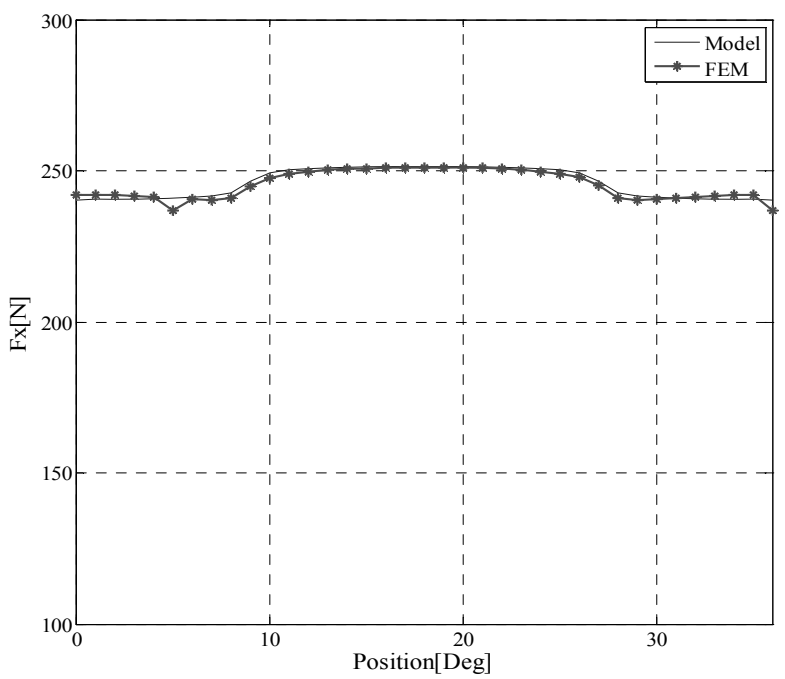

Fig. 6. Suspending force in the $\mathrm{x}$ direction. 


\section{Design of Novel BLSRM}

Based on the above analysis, one $8 / 10$ prototype motor is designed. Fig. 8 shows its stator and rotor. Detailed specifications are shown in Table 1.

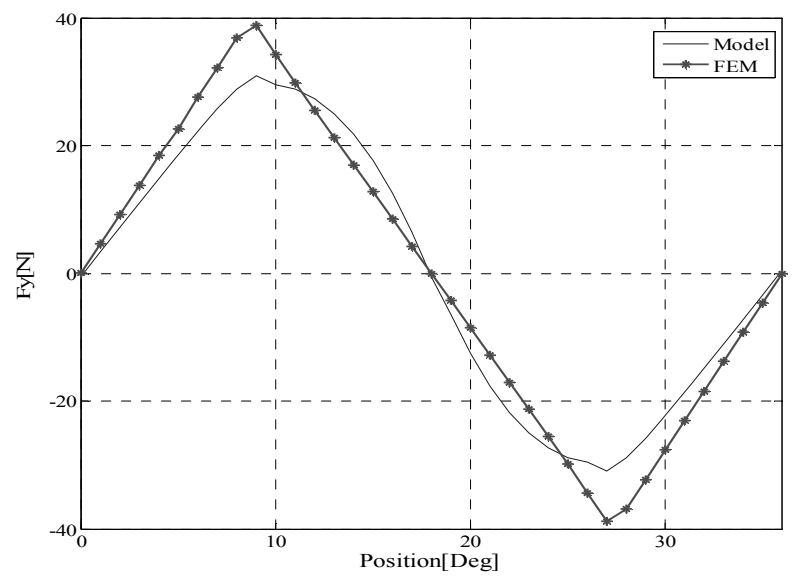

Fig. 7. Suspending force in the y direction.

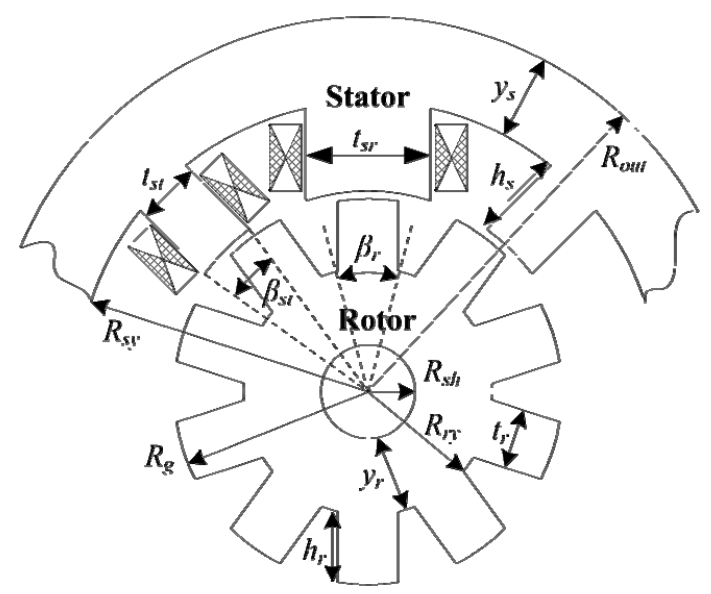

Fig. 8. Dimensions of the proposed BLSRM.

Table. 1. Specifications of prototype BLSRM

\begin{tabular}{c|c}
\hline Parameter & Value \\
\hline Number of stator poles & 8 \\
\hline Number of rotor poles & 10 \\
\hline Pole arc of the stator for torque [deg] & 18 \\
\hline Pole arc of the stator for suspending [deg] & 36 \\
\hline Pole arc of rotor [deg] & 18 \\
\hline Length of axial stack [mm] & 40 \\
\hline Outer diameter of stator [mm] & 112 \\
\hline Inner diameter of stator [mm] & 62 \\
\hline Yoke thickness of stator [mm] & 10 \\
\hline Length of air gap [mm] & 0.3 \\
\hline Inner diameter of rotor [mm] & 18 \\
\hline Yoke thickness of rotor $[\mathrm{mm}]$ & 9.7 \\
\hline
\end{tabular}

Furthermore, according to (14)-(19), the suspending force profiles of stator pole $\mathrm{P}_{\mathrm{x} 1}$ can be obtained as shown in Figs. 9 and 10.

In order to determine the merits of the proposed structure, a conventional BLSRM is also analyzed, as shown in Figs. 11 and 12. From Fig. 9-12, the suspending force in

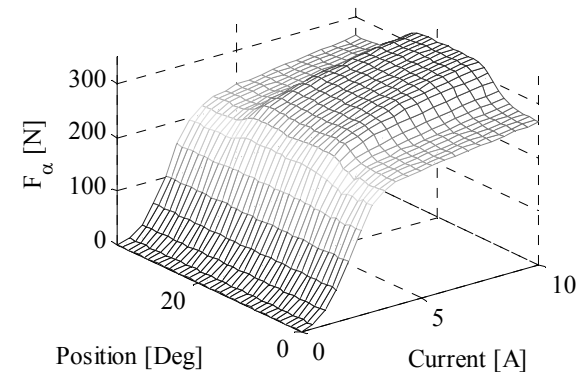

Fig. 9. Suspending force profile of the proposed BLSRM in $\mathrm{x}$ direction.

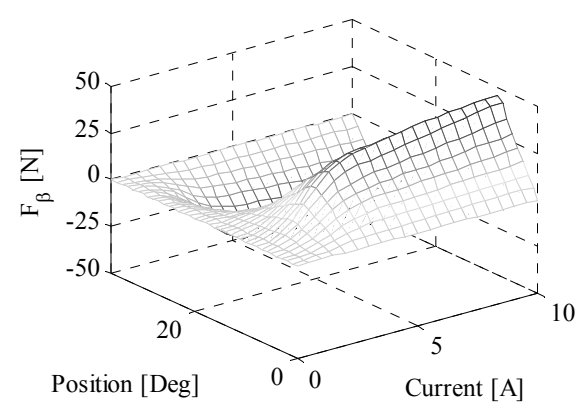

Fig. 10. Suspending force profile of the proposed BLSRM in $\mathrm{y}$ direction.

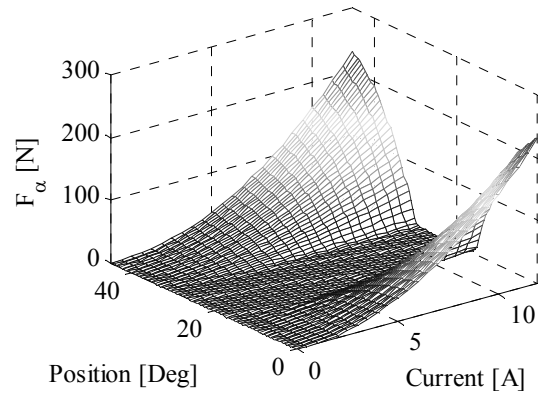

Fig. 11. Suspending force profile of conventional BLSRM in $\mathrm{x}$ direction.

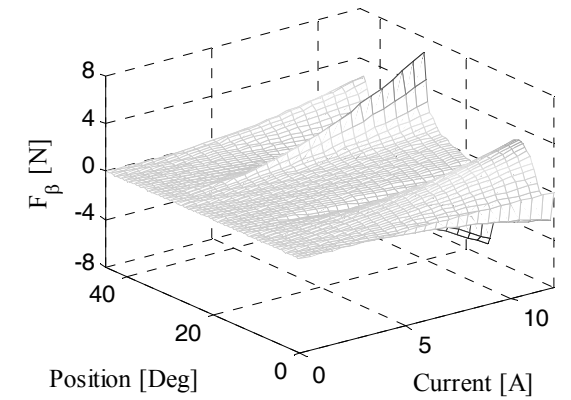

Fig. 12. Suspending force profile of conventional BLSRM in $\mathrm{y}$ direction. 
the proposed structure almost remained constant with the variation in rotor position. However, in the conventional structure, the suspending force clearly varies with the rotor position. Therefore, for conventional BLSRM, the conducting period must be selected in compromise between the torque and suspending force. Moreover, the current has to be increased to generate enough suspending force. This results in complex control scheme and large torque ripple. However, for the proposed BLSRM, much lower current and a simpler control algorithm can be obtained due to the good characteristic of the suspending force.

The torque profile shown in Fig. 13 is good enough to develop torque. The control and drive characteristics are discussed later.

\section{Simulation Results}

To guarantee the steady operation of the BLSRM, the suspending force should be controlled at all positions. Fig. 14 shows the control block diagram.

From Fig. 15, the displacement errors in the two directions are input to the PID controllers to generate suspending force references in such directions. Through the current and the position, actual suspending forces can be obtained by the lookup tables. Two suspending force errors are then input into two hysteresis controllers. Accordingly, a control signal can be generated with some control rules.

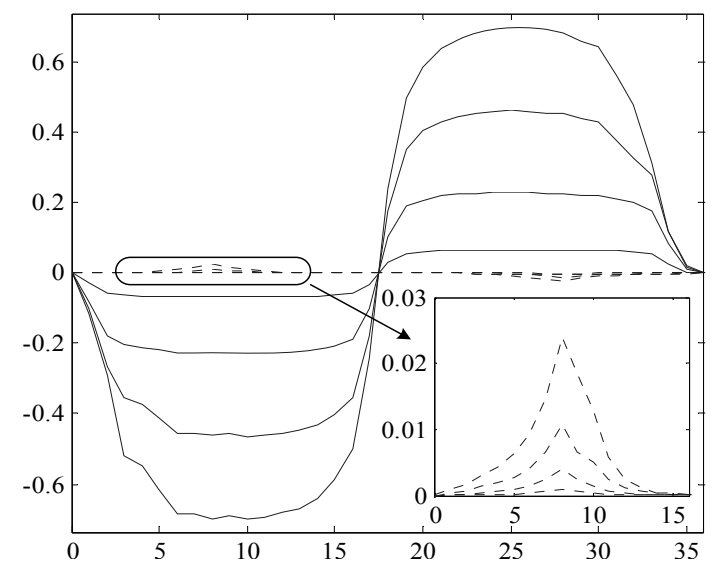

Fig. 13. Torque profiles of the proposed structure.

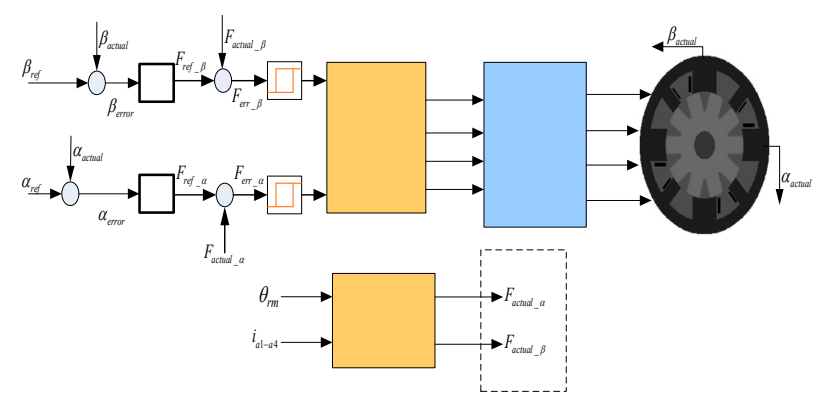

Fig. 14. Block diagram of suspending force control.
In the simulation, the suspending load in the y direction is $10 \mathrm{~N}$ and rotor speed is $1,500 \mathrm{rpm}$. The initial eccentric displacement in the $\mathrm{x}$ and $\mathrm{y}$ directions is $60 \mu \mathrm{m}$ and the current is limited at $10 \mathrm{~A}$. Current $i_{a 1}$ to $i_{a 4}$ are suspending current $i_{1}$ to $i_{4}$, respectively. Figs. 14-18 shows the simulation results.

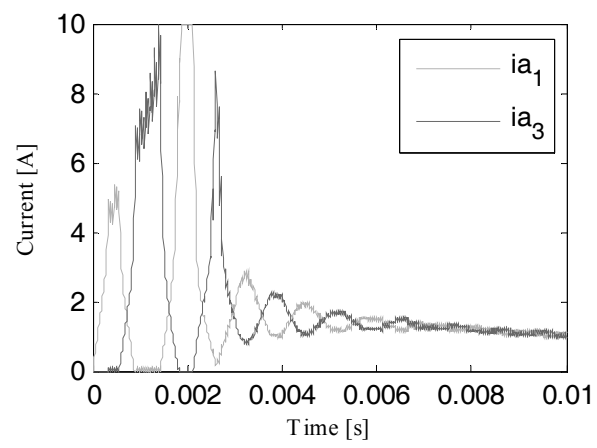

Fig. 15. Suspending currents $i_{1}$ and $i_{3}$.

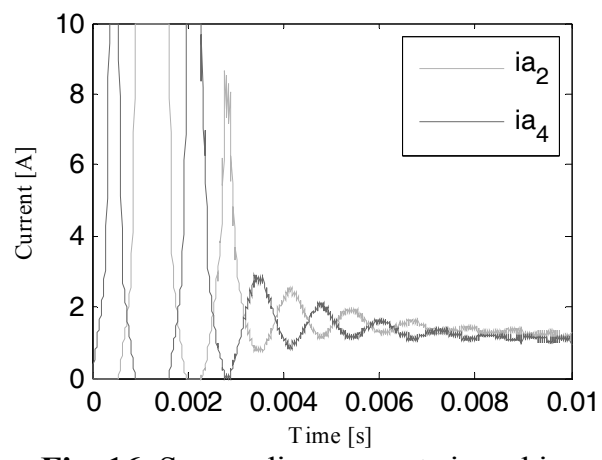

Fig. 16. Suspending currents $i_{2}$ and $i_{4}$.

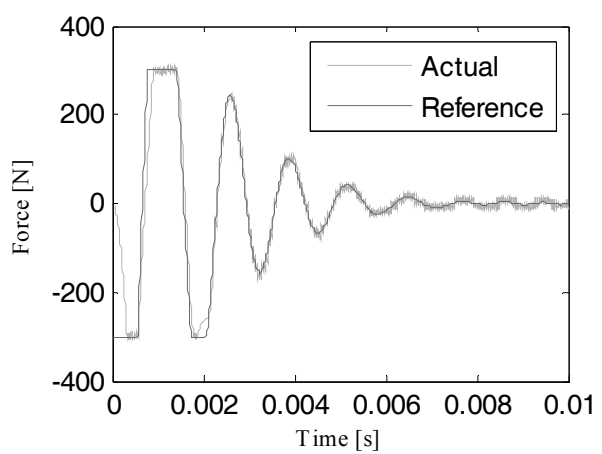

Fig. 17. Suspending force in $\mathrm{x}$ direction.

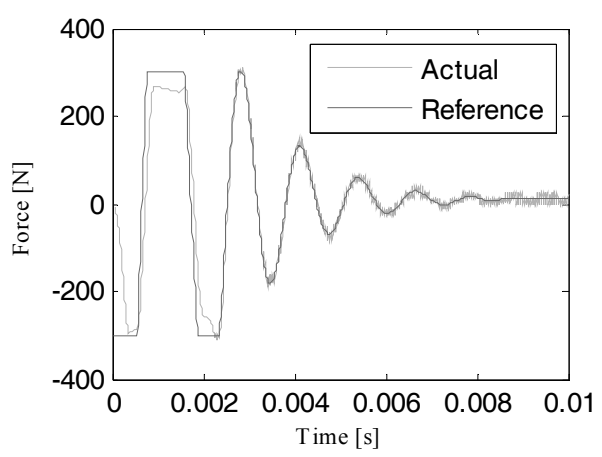

Fig. 18. Suspending force in y direction. 


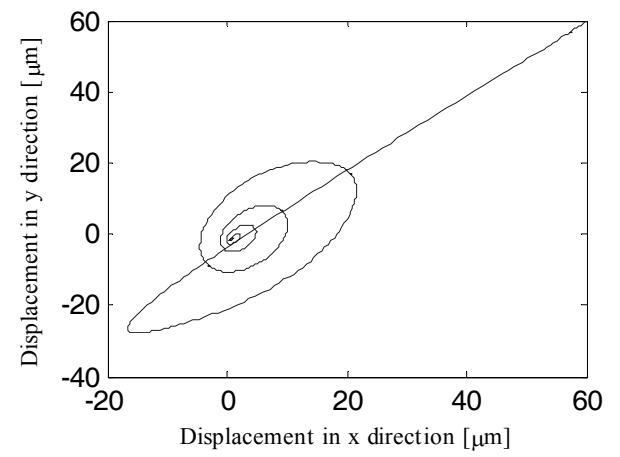

Fig. 19. Rotor locus.

From the simulation results, four suspending currents are independently regulated to control the suspending force in the $\mathrm{x}$ and $\mathrm{y}$ directions. The eccentric error of the rotor can be gradually reduced, as shown in Fig. 19.

\section{Conclusions}

In this paper, a novel structure of BLSRM with hybrid stator poles was proposed. In order to carry out real-time control of BLSRM, a calculation model for suspending force was developed. Compared with conventional BLSRM, the proposed structure has many advantages such as lower thermal load and a simpler control algorithm. The validity of the mathematical model for suspending force was verified by FEM and simulation results.

\section{Acknowledgments}

This work was supported by the National Research Foundation of Korea (NRF) grant funded by the Korean government (Grant No. 2010-0014172)

\section{References}

[1] M. Takemoto, A. Chiba, H. Akagi and T. Fukao, " Suspending Force and Torque of a Bearingless Switched Reluctance Motor Operating in a Region of Magnetic Saturation” in Conf. Record IEEE-IAS Annual Meeting, 2002, pp. 35-42.

[2] L. Chen, W. Hofmann, "Analytically Computing Winding Currents to Generate Torque and Levitation Force of a New Bearingless Switched Reluctance Motor", in Proc.12th EPE-PEMC, Aug, 2006, pp. 1058-1063.

[3] C. R. Morrison. Bearingless Switched Reluctance Motor. U.S. Patent 6,727,618, 2004.

[4] S. Ye. Research on Bearingless Switched Reluctance Motors, Master's thesis, 2003.
[5] Q. Wang, Analysis and Design of Bearingless Switched Reluctance Motor. Master's thesis, 2007.

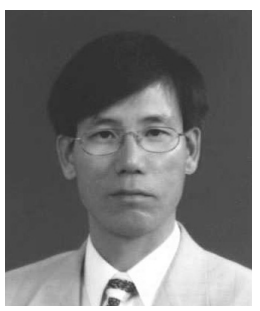

Jin-Woo Ahn was born in Busan, Korea in 1958. He received his B.S., M.S., and Ph.D. degrees in Electrical Engineering from Pusan National University, Pusan, Korea in 1984, 1986, and 1992, respectively.

He has been with Kyungsung University, Busan, Korea as a Professor in the Department of Mechatronics Engineering since 1992. He was a Visiting Researcher in the Speed Lab at Glasgow University, U.K., a Visiting Professor in the Department of ECE and WEMPEC at the University of WisconsinMadison, USA, and in the Department of ECE at Virginia Tech from 2006 to 2007. He was the Director of the Advance Electric Machinery and Power Electronics Center (2004 2008). He has also been the Director of the Smart Mechatronics Advanced Research and Training Center since August 2008 and of the Senior Easy Life Regional Innovation System since July 2008, which are both authorized by the Ministry of Knowledge Economy, Korea. He is the author of five books including SRM and more than 100 research papers, and has several patents. His current research interests are advanced motor drive systems and electric vehicle drives.

Dr. Ahn has received several awards including the Best Paper Award (2002), Academic Achievement Award (2003), Park Min-Ho Special Award (2009) from the Korean Institute of Electrical Engineers, Best Paper Award (2003) from the Korean Federation of Science and Technology, and Academic Achievement Award (2003), Technology Achievement Award (2004), and Best Paper Award (2007) from Korean Institute of Power Electronics. He was also awarded the Best Research Professor (2008), Best Industrial Cooperation Award (2009), and Merit Award (2009) from Kyungsung University. He is a fellow of the Korean Institute of Electrical Engineers, a member of the Korean Institute of Power Electronics, and a senior member of the IEEE.

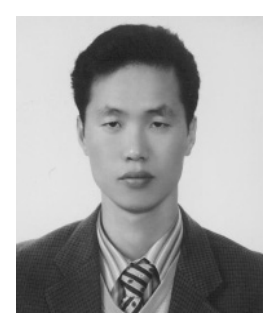

Dong-Hee Lee was born on November 11, 1970 and received his B.S, M.S., and Ph.D degrees in Electrical Engineering from Pusan National University, Busan, Korea in 1996, 1998, and 2001, respectively. He worked as a Senior Researcher for the Servo R\&D Team at OTIS-LG from 2002 to 2005. He has been with Kyungsung University, Busan, Korea as an Assistant Professor in the Department of Electrical and Mechatronics Engineering since 2005. He has been the Director of the Advance Electric Machinery and Power Electronics Center since 2009. His current research interests are servo systems and electrical motor drives with power electronics. 